\title{
MEDICINA CENTRADA EN LA PERSONA: DEL CONCEPTO A LA ACCIÓN
}

\author{
PERSON-CENTERED MEDICINE: FROM CONCEPTTO ACTION
}

\begin{abstract}
Alberto Perales ${ }^{1,2, a}$
Por la década del 60, Carlos Alberto Seguín afirmaba en sus clases sanmarquinas, que la Psiquiatría no era una especialidad médica, sino ciencia básica de la Medicina, y que en todo acto médico el galeno debía Hacer un poco de Psiquiatría, orientada no solo a la enfermedad, sino a la dolencia del paciente como persona. Me permito agregar a ello, que la Psiquiatría constituye la expresión más humana de la vocación médica de servicio, pues lidia no solo con los síntomas del trastorno mental sino, fundamentalmente, con los diversos problemas humanos que contribuyen a su causalidad o surgen como su consecuencia. Es decir, que un adecuado ejercicio de la Medicina obliga a la consideración integral del paciente como "persona en su mundo de circunstancias".
\end{abstract}

En línea coincidente, y a pesar de la distancia espacial y temporal, otro psiquiatra peruano, Juan Enrique Mezzich, entonces presidente de la Asociación Psiquiátrica Mundial, 2005, desarrolla un Programa de Psiquiatría Centrada en la Persona (1). Gradualmente, el éxito que alcanza su propuesta la expande como movimiento programático de medicina centrada en la persona (MCP) al campo general de la salud. Reemerge, así, como indispensable balance equilibrador ante el excesivo reduccionismo científico, influencia deshumanizante de la tecnología médica y participación de intereses económicos de terceras partes que generan un mercado profesional con inmoral concepción de la salud como bien de consumo.

Ulteriormente, con el apoyo de instituciones internacionales clave, la MCP se consolida como movimiento en torno a la creación del Colegio Internacional de Medicina Centrada en la Persona (ICMCP, por sus siglas en inglés) (2) con sede actual en la ciudad de Nueva York. La iniciativa cuenta, además, con una revista internacional, The International Journal of Person Centered Medicine, publicada en colaboración con la Universidad de Buckingham, en la cual se difunden contribuciones significativas de diversas regiones del mundo.

\section{¿QUÉ ES MEDICINA CENTRADA EN LA PERSONA?}

Explicada en forma resumida, la MCP es una doctrina muy antigua y, al mismo tiempo, muy actual. Enfoca al paciente desde una visión y quehacer integrales en todas sus dimensiones humanas, (biológica, psicológica, sociocultural y espiritual), tanto en la atención clínica individual, como en lo referente a la Salud Pública. Aspira, por ello, a ser personalizada, entendiendo a cada paciente como fenómeno único (persona) con sus aspectos de salud negativa (enfermedad y dolencia) y de salud positiva (bienestar y desarrollo personal). Su objetivo final propone integrar el quehacer médico científico y humanista en una trama médico-paciente y equipo de salud-población, con sólido fundamento ético y de impulso al desarrollo humano.

Sus características esenciales han sido resumidas por Mezzich et al.: a) Compromiso ético; b) Marco holístico; c) Sensibilidad y respuesta cultural; d) Foco comunicativo y relacional; e) Individualización del programa de atención clínica; f) Establecimiento de una base común entre clínicos, paciente y familia para el entendimiento y la acción; g) Organización de servicios integrados y centrados en las personas y la comunidad, y h) Educación médica e investigación científica en salud centradas en la persona ${ }^{(3)}$.

Por ello, explorar el devenir de la medicina centrada en la persona en Latinoamérica -con su alta carga de pobreza y subdesarrollo- constituye no solo necesidad científica sino, además, responsabilidad ética, para convertir la atención de salud de nuestra región en cuidado responsable de todo ser humano en situación de enfermedad, y ayudarle al logro del máximo bienestar en condición de salud.

Se comprende, así, la importancia que en la actualidad tiene la MCP para nuestros países como concepto y estrategia que sustenten una atención de salud de mayor calidad científica y humana, en la cual, el respeto a la autonomía, valores y dignidad del paciente constituyan un reconocimiento a su condición de persona como imperativo ético ${ }^{(4)}$.

\section{DESARROLLO DE LA MCPEN EL PERÚ}

En diciembre de 2014, un mes después del segundo congreso Internacional de MCP, llevado a cabo en Argentina, con el tema central "Avanzando en Atención de Salud Humanista e Interdisciplinario" donde se emite la Declaración de Buenos Aires, la Academia Nacional de Medicina del Perú organiza un Encuentro sobre MCP con la participación de

Academia Nacional de Medicina. Lima, Perú

Facultad de Medicina, Universidad Nacional Mayor de San Marcos. Lima, Perú

Editor invitado, medico psiquiatra

Correo electrónico: perales.alberto@gmail.com

Recibido: 04/11/2016 Aprobado: 23/11/2016

Citar como: Perales A. Medicina centrada en la persona: del concepto a la acción [Editorial]. Rev Peru Med Exp Salud Publica. 2016;33(4):605-6. doi: 10.17843/rpmesp.2016.334.2541 
las academias nacionales de medicina de Bolivia, Colombia, Chile y Paraguay y la contribución de diferentes instituciones locales. Se cubre un programa de conferencias y simposios que finalizan con el acuerdo del Acta de Lima. En esta, las cinco academias reconocen el contenido y propuestas de la Declaración de Buenos Aires y proponen organizar una Red Latinoamericana de MCP (RLAMCP) que coordine y difunda los esfuerzos de las academias de medicina, universidades, y otras instituciones de salud de la región, en estrecha colaboración con el International College of Person Centered Medicine. El documento es difundido oficialmente a las cinco academias señaladas y a instituciones locales significativas. Ese mismo año 2014, bajo autoría compartida, se publica en inglés el artículo Latin American Bases and Perspectives on Person Centered Medicine and Health y, en el 2015, en español como "Bases y perspectivas latinoamericanas sobre Medicina y Salud Centradas en la Persona" ${ }^{(5)}$.

En septiembre de 2015 se lleva a cabo, en el marco de las "XIV Jornadas Sanfernandinas" de la Facultad de Medicina de San Fernando de la Universidad Nacional Mayor de San Marcos (Lima, Perú), el "Curso Internacional Investigación y Medicina Centrada en la Persona”. El evento es auspiciado por la Academia Nacional de Medicina y la Red Latinoamericana de Medicina Centrada en la Persona (RLAMCP) y cuenta con la participación de un centenar de profesores de la facultad, de sus escuelas e institutos. En tal evento se ratifica que la ciencia representa un instrumento esencial y el humanismo la esencia de la medicina. En línea con ello, la educación médica y la práctica de la medicina deben estar informadas por la evidencia y la experiencia y orientadas a la totalidad de la persona.

En diciembre del mismo año, la Academia Nacional de Medicina y la RLAMCP, en colaboración con la Universidad Nacional Mayor de San Marcos, la Universidad Peruana Cayetano Heredia, la Universidad Católica Santo Toribio de Mogrovejo y el Instituto Nacional de Salud, organizan, las "Primeras Jornadas Latinoamericanas de MCP: del concepto a la Acción", en Lima, Perú, con el auspicio de las academias nacionales de Medicina de Colombia, Chile y Uruguay, del Colegio Médico del Perú, la Asociación Médica de Brasil, la Organización Panamericana de Salud (OPS) y el International College of Person Centered Medicine. El evento genera la "Declaración de Lima" en cuyas conclusiones, entre otras importantes, se acuerda promover el cultivo de la MCP, sus principios y prácticas en la atención de salud, educación en Medicina y ciencias de la salud, investigación científica y salud pública. Finalmente, organizar encuentros y jornadas de MCP regulares, optimizadas a través de una fluida comunicación bilateral con organizaciones internacionales e International College of Person Centered Medicine ${ }^{(6)}$.

De esta reunión, se publica como simposio en el presente número: "Medicina Centrada en la Persona: del concepto a la acción" que incluye algunos de los trabajos discutidos en tal ocasión, como, por ejemplo: Sofía Cuba y Zoila Romero destacan, en su trabajo sobre "El método clínico centrado en la persona y su aplicación en la Atención Primaria de Salud", la importancia del método clínico centrado en el paciente en el contexto de un sistema de atención primaria de salud basado en las necesidades de la comunidad.

Javier E. Saavedra y Verousckha Uchofen-Herrera, en su trabajo "Percepciones sobre la atención de salud en personas con problemas autoidentificados de salud mental en zonas rurales del Perú", analizan, con metodología cualitativa, las percepciones de 235 sujetos con problemas de salud mental (PSM) de localidades rurales de la costa, sierra y selva del Perú, sobre causalidad, recursos personales y expectativas de atención. Encuentran asociación entre tales problemas y conflictos familiares y/o pérdida de seres significativos, aunque un grupo importante de los probandos desconocen qué esperan de los establecimientos de salud.

Juan Enrique Mezzich y Alberto Perales, en su artículo "Atención clínica centrada en la persona: principios y estrategias", luego de resumir los conceptos esenciales de la MCP, revisan estrategias evaluativas de tales principios en la aplicación práctica de los sistemas integrados de atención de salud subrayando la importancia de crear instrumentos adaptados a tales fines proponiendo, como ejemplo, el índice de atención centrada en la persona (PCI).

Alberto Perales, Juan Mendoza, Rodolfo Armas y Oscar Cluzet, de las academias de medicina de Perú, Colombia, Chile y Uruguay, emiten opinión sobre aspectos de concepto, necesidad y desarrollo de la MCP, enlazándolos con las características, históricas y actuales del ejercicio profesional de la medicina en sus respectivos países, que justifica el título de sus aportes: "Perspectivas latinoamericanas sobre medicina centrada en la persona".

Finalmente, en el marco del subtítulo de esta editorial, considero, desde una óptica personal, que el fundamento epistemológico de la MCP genera un reclamo de acción que desborda los específicos campos de la medicina y de la salud, exigiendo que todos los seres humanos debiéramos tratar y ser tratados como personas, en cualquier nivel de interacción social.

Percibo así, su doctrina como una propuesta o estrategia moral de bien común, de paz, lograda por el trato justo a todo ser humano como fin y no como medio. De aceptar tal reflexión como probable, arribaríamos también a una conclusión práctica: nadie puede dar lo que no tiene. Para tratar al otro como persona y formar a otros como personas debemos, primero, nosotros los médicos y todos los profesionales de la salud, aprender a ser PERSONAS y cultivarnos permanentemente como tales en una búsqueda sin fin de trascendencia.

\section{REFERENCIAS BIBLIOGRÁFICAS}

1. Mezzich JE. Psiquiatría orientada a la persona: articulación de la ciencia y el humanismo de la medicina. World Psychiatry (Edición en español). 2007; 5(2):65-7.

2. Mezzich JE, Appleyard J, Botbol M, Ghebrehiwet T, Groves J, Salloum I, et al. International Journey and the Development of Person Centered Medicine (Editorial Introduction) The International Journal of Person Centered Medicine. 2014; 4(4): 211-16
3. Mezzich JE. Repensando el Centro de la Medicina: De la enfermedad a la persona. Acta méd. Per. 2010; 27(2): 148-150.

4. Fulford KWM, George N. Christodoulou GN, Stein DJ. Values and Ethics: Perspectives on Psychiatry for the Person. The International Journal of Person Centered Medicine. 2011;1 (1):161-62.

5. Wagner P, Perales A, Armas R, Codas O, de los Santos R, Elio-Calvo D, et al. Latin
American Bases and Perspectives on Person Centered Medicine and Health. The International Journal of Person Centered Medicine. 2014; 4 (4): 220-7.

6. Latin American Network for Person Centered Medicine (2016). The 2015 Lima Declaration on Latin American Developments for Person Centered Medicine. International Journal of Person Centered Medicine, 6: 7-8. 